\title{
Cytogenetic analysis of early pregnancy loss after assisted reproduction treatment using intracytoplasmic sperm injection
}

\author{
Aret Kamar ${ }^{1} \mathbb{D}$, Nurettin Turktekin ${ }^{1} \mathbb{D}$, Ramazan Ozyurt ${ }^{1} \mathbb{D}$, Cemil Karakus ${ }^{2} \mathbb{D}$, \\ Devrim Saribal ${ }^{3}$, , F. Sinem Hocaoglu-Emre ${ }^{4}$ (D) \\ ${ }^{1}$ Istanbul Center for Assisted Reproduction and Gynecology, Istanbul, Turkey \\ ${ }^{2}$ Clinic Nişantaşı, Istanbul, Turkey \\ ${ }^{3}$ Istanbul University-Cerrahpaşa, Cerrahpaşa Medical School, Department of Biophysics, Istanbul, Turkey \\ ${ }^{4}$ Beykent University, Department of Nutrition and Dietetics, Istanbul, Turkey
}

\begin{abstract}
Objectives: To evaluate the incidence of numerical chromosomal abnormalities in the patients with early pregnancy loss (EPL) following in vitro fertilization, and evaluate the role of different confounders of the risk of chromosomal abnormality-related pregnancy loss.

Material and methods: A retrospective chart review of all patients from our in vitro fertilization (IVF) center who conceived using assisted reproduction techniques between April 2017 and 2019, who experienced a subsequent early pregnancy loss, and whose abortus materials were successfully karyotyped were included.

Results: Of the 243 patients experienced an early loss, the overall rate of chromosomal abnormality was $46.75 \%$. The overal rate of aneuploidy in our patient group was $88.8 \%(64 / 72)$, whereas $6.94 \%(5 / 72)$ of the abnormal karyotypes were polyploid. The most common type of trisomy was Trisomy 16 (20.0\%; 11/55) followed by Trisomy 15 (14.5\%; 8/55). Univariate and multivariate analyses showed that maternal age ( $<35$ years) and the total number of retrieved oocytes per cycle $(\geq 5)$ were risk factors for a chromosomal abnormality $(<0.001 ;<0.05$, respectively). The adjusted OR of karyotypic abnormalities was 0.45 for the antagonist cycle type $(p<0.05)$, and 0.58 for frozen embryo transfer $(p<0.05)$.

Conclusions: Karyotypic abnormality is one of the main reasons for pregnancy loss following an IVF procedure. Although the pregnancy rates increased as a result of novel technologies, the ratio of EPL is still high. The implementation of preimplantation genetic screening techniques might lower the incidence of EPL due to chromosomal abnormalities, thus decreasing the burden on the physicians and the patients.
\end{abstract}

Key words: abortus; early pregnancy loss; assisted reproduction; ICSI; cytogenetic analysis

Ginekologia Polska 2021; 92, 7: 475-480

\section{INTRODUCTION}

Early pregnancy loss (EPL) is the most frequent pregnancy-related complication and accounts for $10-20 \%$ of clinically confirmed pregnancies. Most of the cases occur before the 20 weeks of gestational age, and up to $50 \%$ of them are related to chromosomal abnormalities [1].

Although the wide use of assisted reproductive technology (ART) improved the pregnancy ratio especially in cases with higher maternal age, EPL is still common following ART due to numerous causes including medication administered during the process, the technique used, and various patient-related factors [2]. The etiologies underlying the pathogenesis of EPL in the cases that underwent ART procedures are different from the ones that conceived naturally [3]. Applying for an ART procedure increases the expectation of pregnancy for the patients despite the increased rate of EPL in this group, and the analysis of possible causes and taking measures for prevention are of concern in order to minimize the psychological and economic burden for the patients. Being the most common cause of EPL in ART cases, chromosomal abnormalities include mostly numerical abnormalities, mosaicisms, and structural chromosomal defects involving autosomal and/or gonosomal chromosomes [4].

The present retrospective study aims to explore the effect of different patient- and procedure-related variables on the risk of chromosomal abnormalities in cases that underwent ART and experienced an EPL. 


\section{MATERIAL AND METHODS}

We performed a cohort of 243 spontaneous miscarriages of a specific institution specialized in ART and in vitro fertilization (IVF) techniques between April 2017 and 2019. Ethical approval was obtained from the institutional board. Pregnancies occurred using intracytoplasmic sperm injection (ICSI) and experienced an early pregnancy loss were scanned retrospectively, and among these, 235 were karyotyped successfully. Exclusion criteria included: abnormal karyotype of one parent, spontaneous pregnancy with the induction, intrauterine insemination (IUI), multiple pregnancies, inadequate fetal tissue for analysis, and lack of the consent for karyotyping.

Of the resulting 154 nonviable embryos, 72 with abnormal karyotype acted as the outcome group and compared to the nonviable embryo group $(n=82)$ with normal karyotyping results in terms of variables including basic demographic information, procedure-related factors such as a previous history of frozen embryo transfer (FET) cancellation, type of the protocol administered for ovarian stimulation, total oocytes retrieved implanted embryo type, sperm abnormality, the gestational week at the time of EPL, endometrial thickness and serum hormone levels. The oocyte output rate was calculated using the number of oocytes retrieved/AFCx $100 \%$ formula. Informed consent was obtained from each patient, and they were informed that their clinical data could be used for research purposes.

\section{IVF treatment}

Of the 154 women, 37 women were diagnosed with unilateral or bilateral tubal obstruction. The male partners of 73 women were diagnosed with azoospermia or oligoasthenoteratozoospermia. Sixteen couples had infertility-causing sperm and hysterosalpingography (HSG) abnormalities in both females and males. The remaining 60 cases were diagnosed with idiopathic infertility.

Natural, antagonist, and semi-antagonist protocols were performed for ovarian stimulation. Controlled ovarian stimulation was performed using recombinant FSH (rec-FSH) and $\mathrm{GnRH}$ antagonist agents as previously described [5]. The dose of the stimulating agent was determined based on the woman's serum hormone levels, age, BMI, and other patient-related characteristics according to the performing gynecologist's personal experience. $10.000 \mathrm{IU}$ of human chorionic gonadotropin ( $\mathrm{hCG}$ ) was administered when more than two follicles reached a diameter of $18-20 \mathrm{~mm}$. After 32-35 hours of induction, oocyte collection was performed through transvaginal ultrasound-guided aspiration.

All the participants in the study group received intracytoplasmic sperm injection (ICSI). After the transfer of Day-3, Day-4, or Day-5 embryos, serum beta-hCG levels were measured on the $12-14^{\text {th }}$ day of the embryo transfer and were accepted as a positive result for the values $\geq 10 \mathrm{IU}$. Clinical pregnancy was confirmed when the intrauterine gestational sac was detected, and the fetal heartbeat was present on the transvaginal USG at 21 days after the transfer. The diagnosis of EPL was confirmed with transvaginal ultrasonography, and curettage of the uterine cavity was performed afterward. Collected samples were routinely sent to the genetic diagnosis center for karyotype analysis. Chorionic villi samples were obtained cautiously in order to avoid maternal contamination. Samples were subjected to karyotype analysis after culture, harvesting, and G-banding. More than 20 cells in metaphase were examined and reported.

\section{Statistical analysis}

All data were stored in a computerized database. Statistical calculations of the data were performed using the SPSS software package program, version 12.0 for Windows. The comparison of the variables between two subgroups was done using 2-tail Student's t-test for independent samples. A Chi-square test was performed when appropriate. Univariate and multivariate analysis were used to determine potential risk factors and calculate the adjusted ORs for chromosomal abnormalities. Quantitative data were presented as mean \pm standard deviation (SD), and risk factors were reported as odds ratio (OR) and $95 \%$ confidence interval (Cl). A p-value $<0.05$ was considered statistically significant.

\section{RESULTS}

The mean age of patients was $37.66 \pm 4.86$ in the abnormal karyotype group and $33.50 \pm 5.98$ in the normal karyotype group. The mean gestational age was $8.55 \pm 1.69$ weeks (median: 8.0 weeks) in the chromosomal abnormality group versus $9.07 \pm 3.37$ weeks (median: 8.0 weeks) in the normal karyotype group. In $94.63 \%$ of cases, the pregnancy terminated before the 12th week of gestation. Overall cytogenetic results revealed that $46.75 \%(72 / 154)$ of the miscarriages showed an abnormal karyotype.

The male to female ratio of the embryos was 1:1.8 (55/99), and the ratio of karyotypic abnormalities in male and female embryos was $56.3 \%$ and $41.4 \%$, respectively. The overall rate of aneuploidy in our patient group was $88.8 \%$ (64/72), whereas $6.94 \%(5 / 72)$ of the abnormal karyotypes were polyploid. The most common type of trisomy was Trisomy 16 (20.0\%; 11/55) followed by Trisomy 15 (14.5\%; 8/55). Three patients had monosomy $X$. Double aneuploidy was present in five cases.

The baseline characteristics of the groups with normal and abnormal karyotype were summarized in Table 1. The frequency of abortus was $70.12 \%$ (108/154), and abnormal karyotype was $61.11 \%(44 / 72)$ in the cases who received frozen embryo transfer. Microdeletions or microduplications were identified in six cases $(8.33 \%$ of the identified 


\begin{tabular}{|l|l|l|l|}
\hline \multicolumn{2}{|l|}{ Table 1. Comparison of the characteristics of early pregnancy loss cases with or without a fetal chromosome abnormality } \\
\hline & Chromosomal Abnormality (n= 72) & Controls (n= 82) \\
\hline Maternal age [years] & $37.66 \pm 4.86$ & $33.55 \pm 5.98$ \\
\hline BMI & $26.42 \pm 5.09$ & $26.21 \pm 4.93$ \\
\hline Total number of previous pregnancy loss & $0.56 \pm 0.94$ & $0.41 \pm 0.83$ \\
\hline Endometrial Thickness & $10.66 \pm 2.05$ & $10.32 \pm 1.98$ \\
\hline Oocyte output rate & $111.81 \pm 66.70$ & $125.27 \pm 74.20$ \\
\hline Total number of oocytes retrieved & $11.19 \pm 10.52$ & $17.32 \pm 12.74$ \\
\hline AMH level (ng/mL) & $1.91 \pm 3.24$ & $2.97 \pm 2.42$ \\
\hline Estradiol level at the time of HCG administration $(\mathrm{pg} / \mathrm{mL})$ & $2417 \pm 2300$ & $3981 \pm 3634$ \\
\hline Progesterone level at the time of oocyte collection $(\mathrm{ng} / \mathrm{mL})$ & $0.82 \pm 0.86$ & 0.24 \\
\hline Gestational week at the time of loss & $8.55 \pm 1.69$ & $1.25 \pm 1.20$ \\
\hline
\end{tabular}

$\mathrm{BMI}$ — body mass index; $\mathrm{AMH}$ - anti-Mullerian hormone

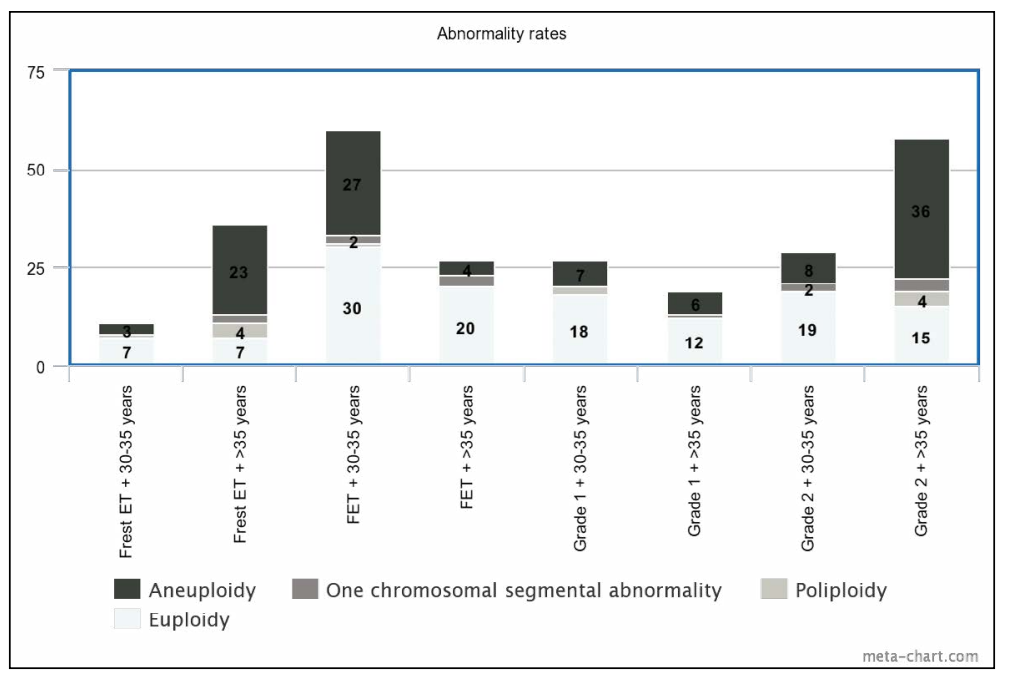

Figure 1. Distribution of the chromosomal abnormalities among different patient characteristics

abnormalities), including three duplications, one deletion, and two complex abnormalities. The distribution of the chromosomal abnormalities among different patient characteristics was shown in Figure 1.

The frequency of abnormal karyotypes in abortus cases was significantly higher, while the maternal age was $>35$ years. There was a statistically significant difference in the maternal age, baseline anti-Mullerian hormone (AMH) value, estradiol, and progesterone levels at the time of oocyte collection, total oocytes retrieved between the two groups. The BMI values, the total number of pregnancy loss, the number of FET cancellation, endometrium thickness, number of women with previous pregnancy loss, oocyte output rates were similar between the groups. The results from the univariate and multivariate regression analyses were summarized in Table 2. Univariate and multivariate analyses showed that maternal age ( $<35$ years) and the total number of retrieved oocytes per cycle $(\geq 5)$ were risk factors for a chromosomal abnormality $(<0.001 ;<0.05)$. The adjusted OR of karyotypic abnormalities was 0.45 for the antagonist cycle type $(p<0.05)$, and 0.58 for frozen embryo transfer $(p<0.05)$.

\section{DISCUSSION}

$\mathrm{EPL}$ is the most common pregnancy-related complication and up to $20 \%$ of clinically confirmed pregnancies end within the first trimester. Although the etiology underlying $\mathrm{SA}$ is highly complex and including various genetic, organic, and systemic factors, the presence of a chromosomal abnormality is the most frequently observed aspect in the pathogenesis. Among them, numerical abnormalities, mainly trisomies are the most widely observed pathologies [6].

The success of an ART procedure depends on the ratio of live birth, and identification of factors and evaluation of possible reasons causing an undesirable outcome is crucial. In the present study, data from multiple linear regression analysis showed that maternal age and oocyte numbers were independent predictors of chromosomal abnormality in the patients who underwent IVF treatment and experi- 
Table 2. Univariate and multivariate analysis of factors related to abortus with chromosomal abnormality

\begin{tabular}{|c|c|c|c|c|c|c|}
\hline & $\begin{array}{l}\text { Chromosomal } \\
\text { Abnormality }(n=72)\end{array}$ & $\begin{array}{l}\text { Controls } \\
(n=82)\end{array}$ & $\begin{array}{l}\text { Crude OR } \\
(95 \% \mathrm{Cl})\end{array}$ & $p$ value & $\begin{array}{l}\text { Adjusted OR } \\
(95 \% \mathrm{Cl})\end{array}$ & p value \\
\hline \multicolumn{7}{|l|}{ Age [years] } \\
\hline$<30$ & 4 & 18 & \multicolumn{4}{|c|}{ Baseline variable } \\
\hline $30-35$ & 17 & 37 & $\begin{array}{l}1.73 \\
(0.60-7.04)\end{array}$ & NS & $\begin{array}{l}1.69 \\
(0.48-4.32)\end{array}$ & NS \\
\hline$>35$ & 51 & 27 & $\begin{array}{l}2.15 \\
(2.61-27.65)\end{array}$ & $<0.0001$ & $\begin{array}{l}1.84 \\
(2.02-19.54)\end{array}$ & $<0.001$ \\
\hline \multicolumn{7}{|l|}{ DET cancellation } \\
\hline No & 54 & 65 & \multicolumn{4}{|c|}{ Baseline variable } \\
\hline Yes & 18 & 17 & $\begin{array}{l}1.20 \\
(0.59-2.71)\end{array}$ & NS & $\begin{array}{l}0.98 \\
(0.36-1.86)\end{array}$ & NS \\
\hline \multicolumn{7}{|c|}{ Sperm Abnormality } \\
\hline No & 37 & 34 & \multicolumn{4}{|c|}{ Baseline variable } \\
\hline Yes & 35 & 38 & $\begin{array}{l}0.92 \\
(0.44-1.62)\end{array}$ & NS & $\begin{array}{l}0.78 \\
(0.27-1.14)\end{array}$ & NS \\
\hline \multicolumn{7}{|c|}{ Endometrial Thickness } \\
\hline $8 \mathrm{~mm}$ & 54 & 71 & \multicolumn{4}{|c|}{ Baseline variable } \\
\hline$\leq 8.0 \mathrm{~mm}$ & 17 & 11 & $\begin{array}{l}1.78 \\
(0.87-4.69)\end{array}$ & NS & $\begin{array}{l}0.93 \\
(0.46-2.44)\end{array}$ & NS \\
\hline \multicolumn{7}{|l|}{ Cycle type } \\
\hline Natural & 27 & 14 & \multicolumn{4}{|c|}{ Baseline variable } \\
\hline Antagonist & 40 & 62 & $\begin{array}{l}0.59 \\
(0.15-0.71)\end{array}$ & $<0.001$ & $\begin{array}{l}0.45 \\
(0.13-0.58)\end{array}$ & $<0.05$ \\
\hline Semi-natural & 5 & 6 & $\begin{array}{l}0.97 \\
(0.11-1.66)\end{array}$ & NS & $\begin{array}{l}0.88 \\
(0.07-1.12)\end{array}$ & NS \\
\hline \multicolumn{7}{|c|}{ Total oocytes retrieved } \\
\hline$\leq 5$ & 26 & 15 & $\begin{array}{l}1.56 \\
(0.88-4.25)\end{array}$ & $<0.05$ & $\begin{array}{l}1.27 \\
(0.76-3.57)\end{array}$ & $<0.05$ \\
\hline $6-20$ & 34 & 38 & \multicolumn{4}{|c|}{ Baseline variable } \\
\hline$\geq 21$ & 11 & 28 & $\begin{array}{l}0.53 \\
(0.19-1.01)\end{array}$ & $<0.01$ & $\begin{array}{l}0.51 \\
(0.17-0.89)\end{array}$ & $<0.05$ \\
\hline \multicolumn{7}{|c|}{ Transferred embryo type } \\
\hline Fresh & 28 & 18 & \multicolumn{4}{|c|}{ Baseline variable } \\
\hline Frozen & 44 & 64 & $\begin{array}{l}0.66 \\
(0.21-0.89)\end{array}$ & $<0.05$ & $\begin{array}{l}0.58 \\
(0.17-0.74)\end{array}$ & $<0.05$ \\
\hline
\end{tabular}

enced an EPL. Additional confounders DET cancellation, presence of a sperm abnormality, endometrial thickness had no impact on the chromosomal abnormality risk. We also found that there was a significant difference between the two groups in terms of AMH levels, E2 and P4 levels at the time of oocyte collection, the total number of collected oocytes whereas, the total number of previous pregnancy loss, body mass index (BMI), endometrial thickness, number of patients with a previous pregnancy loss did not differ.

In their large case series of 676 spontaneous miscarriages following ART protocols, Martinez et al. reported that the $51.88 \%$ of the miscarriages after ART and $51.82 \%$ conceived by ICSI had an abnormal karyotype with mostly autosomal trisomies, among which, trisomy of the chromosome 16 was the most observed genotype, which is consistent with our findings [6]. It has been suggested that trisomies develop during oogenesis, and acrocentric' chromosomes 13-15, 21, and 22 were the most affected ones as a result of failures during the meiosis, especially for the oocytes stayed in meiosis I phase for up to 40 years [7]. The frequency of triploidy was $6.94 \%$ in our group, which was relatively higher than the previous reports [8, 9]. Polyploidy was observed in the abortus material obtained from women with a mean age of 38.4 (ranging between 3244 years) possibly due to increased chromosomal nondisjunction and improper spindle formation during meiosis as a result of advanced maternal age. The probability of dispermy might be excluded since the records of the IVF procedure under the microscope did not reveal any sign of the incident. 
It has been shown that $\mathrm{AMH}$ levels are associated with the implantation rates following ART procedures [10, 11]. In our series, we observed that the AMH levels and the total number of collected oocytes in the cycle were significantly lower in the chromosomal abnormality group. This might be a result of age-related decline in the levels of the hormone and ovarian reserve, and age-related increase in chromosomal abnormality rates. Thus, AMH levels could not be suggested as an indicator of possible chromosomal abnormality-related EPL. Similarly, the levels of E2 and P4 were also lower in the chromosomal abnormality group, possibly due to age-dependent factors. It has been shown that aneuploidy ratio was significantly higher in women older than 35 years old and with lower AMH levels, even after age stratification, suggesting that age-related changes in the oocytes rather than diminished ovarian reserve might be causing the embryonal aneuploidy [12].

Endometrial thickness was suggested to be an indicator of pregnancy outcome, and birth rates were shown to be higher when the endometrial thickness was above a limit of $8 \mathrm{~mm}$ [13]. In our study group, we did not observe a significant difference between the two groups in terms of endometrial thickness. Additionally, when we sub-grouped patients in terms of endometrial thickness and chromosomal abnormality, the multivariate analysis did not yield a significantly increased risk for this measure.

The implantation rates were shown to be higher after frozen single blastocyst transfer, whereas the ratio of pregnancy loss after frozen and fresh single blastocyst transfer was similar [14]. In our cases, we found that the relative risk of chromosomal abnormality was significantly lower in the frozen embryo group. Consistent with our findings, Wu et al. found a higher ratio of chromosomal aberration in the cases who underwent fresh ET procedure in their study of EPL cases conceived using different ART [15]. Freezing and elective transfer of embryos might be a factor in relatively lower risk, eliminating the endocrinological endometrium related perturbations.

The ratio of female fetuses among the abortus cases was significantly higher in our series in accordance with the previous data. Although the presence of a gender-related mechanism underlying these results has not been revealed yet, there are several possible suggestions including discrepancies during the intrauterine inactivation of X-chromosome and presence of $X$-chromosomal recessive lethal mutations $[16,17]$. However, it has been reported that maternal cell contamination can be the result of a higher female ratio in abortus materials, and the ratio of maternal contamination was shown to be up to $22 \%$ by a recent single-nucleotide polymorphism chromosomal array analysis study [18, 19].

When compared to the comprehensive worldwide data collected from the abortus cases conceived by ICSI, the ratio of fetuses with abnormal karyotyping results is slightly lower in our study group, suggesting that might be a cause of our extended exclusion criteria [9, 20]. However, the karyotyping method used for the screening of aborted embryos might be underestimating a proportion of the cases. Although the karyotyping technique used in the context of this study is a conventional golden standard method, it still bears several limitations including lower resolution limited to larger chromosome segments, therefore missing microdeletions, microduplications and subtle rearrangements, and insufficiency in detecting mosaic cases, especially when low-level mosaicism is present [21]. Although novel technologies as array-based comparative genomic hybridization (aCGH), and next-generation sequencing technologies are recommended for the detection of chromosomal abnormalities in the SA cases in order to provide better accuracy, the cost of the methods is relatively high [22].

Revealing the presence of a chromosomal aberration underlying the $\mathrm{SA}$ is of utmost importance to provide a better pregnancy outcome for the patient and future cases, yet, the etiology for almost half of the cases remains unclear. A recent meta-analysis by Robbins et al. reported that pathogenic variants of the genes clustered in the pathways related to gene expression, embryonic development, mitosis, and cell cycle progression, and inflammation and immunity might be causing non-aneuploid early pregnancy loss [23]. However, these genes can not be scanned through conventional methods and preimplantation genetic detection (PGD) techniques, and meanwhile, the routine implementation of the investigation is impractical. Implementation of a PGD procedure during the ART process is related to a lower rate of miscarriage among women between 35-37 years old, and a higher rate of a live-birth among women $>37$ years old [24]. Although $P G D$ is an alternative method that might be introduced to reduce the EPL rates and prevent implantation of chromosomal abnormality carrying embryos, hence improving the clinical prognosis, it is not demanded by most of the couples due to its higher cost. Furthermore, current PGD methods can only detect copy number gain or losses, and if present, some triploidy, and tetraploidy cases will still remain undetected [25].

The strengths of this study present the data derived from elective single embryo transfer cases that allow to rule out the embryo related factors. As parental confounders might be a risk factor for fetal chromosomal abnormality-related abortus, we excluded the individuals with any maternal or paternal abnormal karyotype that can contribute to chromosomal abnormalities. Additionally, we meticulously embraced the included cases among the pregnancy loss data of our center. The ratios of chromosomal abnormalities, abnormality types, and the chromosomes involved in trisomy cases were well in agreement with existing literature and might be a reflection of the clinical practice. Laboratory and 
technical equipment, conditions, staff, and applied protocols were the same in all cases. However, there are limitations to this study. We did not subgroup the cases according to the day of embryo transfer due to a small number of patients per group, making the statistical evaluation difficult; thus the possible effects of culture medium related factors and incubation duration might be undervalued.

\section{CONCLUSIONS}

The present study shows that karyotypic abnormality is one of the main culprits for EPL, with an increasing rate as a result of maternal age and other defined confounders. Of the chromosomal alterations, aneuploidy was the most detected abnormality type in our series. Although recent advances in ART improved the pregnancy rates in patients with different infertility etiologies, the ratio of EPL following ART protocols still did not deteriorate to desirable levels. Increasing the ratio of live birth after ART treatment is the expected outcome, and further studies and reports of different series are needed in order to understand the underlying etiology of EPL and prevent chromosomal abnormality-related losses, and possible birth defects. Herein, we suggest that PGD using sensitive methods is necessary notably for women older than the age of 35 years in order to prevent EPL incidents of ART pregnancy, thus prevent and lower the physiological, psychological, and economic burden.

\section{Conflict of interest}

The authors declare no conflict of interest.

\section{REFERENCES}

1. Spontaneous Abortion. 1992, doi: 10.1007/978-1-4471-1918-0.

2. Balen AH, MacDougall J, Tan SL. The influence of the number of embryos transferred in 1060 in-vitro fertilization pregnancies on miscarriage rates and pregnancy outcome. Hum Reprod. 1993; 8(8): 1324-1328, doi: 10.1093/oxfordjournals.humrep.a138250, indexed in Pubmed: 8408536.

3. Westergaard HB, Johansen AM, Erb K, et al. Danish National IVF Registry 1994 and 1995. Treatment, pregnancy outcome and complications during pregnancy. Acta Obstet Gynecol Scand. 2000; 79(5): 384-389, indexed in Pubmed: 10830766.

4. lews M, Tan J, Taskin O, et al. Does preimplantation genetic diagnosis improve reproductive outcome in couples with recurrent pregnancy loss owing to structural chromosomal rearrangement? A systematic review. Reprod Biomed Online. 2018; 36(6): 677-685, doi: 10.1016/j. rbmo.2018.03.005, indexed in Pubmed: 29627226.

5. Ubaldi FM, Capalbo A, Colamaria S, et al. Reduction of multiple pregnancies in the advanced maternal age population after implementation of an elective single embryo transfer policy coupled with enhanced embryo selection: pre- and post-intervention study. Hum Reprod. 2015; 30(9): 2097-2106, doi: 10.1093/humrep/dev159, indexed in Pubmed: 26150408.

6. Martínez MC, Méndez C, Ferro J, et al. Cytogenetic analysis of early nonviable pregnancies after assisted reproduction treatment. Fertil Steril. 2010; 93(1): 289-292, doi: 10.1016/j.fertnstert.2009.07.989, indexed in Pubmed: 19748088.

7. Ou Z, Yin M, Chen Z, et al. Meta-analysis of the association between chromosomal polymorphisms and outcomes of embryo transfer following in vitro fertilization and/or intracytoplasmic sperm injection. Int J Gynaecol Obstet. 2019; 144(2): 135-142, doi: 10.1002/ijgo.12702, indexed in Pubmed: 30378097.

8. Rodriguez-Purata J, Lee J, Whitehouse M, et al. Embryo selection versus natural selection: how do outcomes of comprehensive chromosome screening of blastocysts compare with the analysis of products of conception from early pregnancy loss (dilation and curettage) among an assisted reproductive technology population? Fertil Steril. 2015; 104(6): 1460-14666.e1, doi: 10.1016/j.fertnstert.2015.08.007, indexed in Pubmed: 26361205.

9. Nayak S, Pavone ME, Milad M, et al. Aneuploidy rates in failed pregnancies following assisted reproductive technology. J Womens Health (Larchmt). 2011;20(8): 1239-1243, doi: 10.1089/jwh.2010.2648, indexed in Pubmed: 21732812

10. Revelli A, Biasoni V, Gennarelli G, et al. IVF results in patients with very low serum AMH are significantly affected by chronological age. J Assist Reprod Genet. 2016; 33(5): 603-609, doi: 10.1007/s10815-016-0675-7, indexed in Pubmed: 26888025.

11. Alanazi $\mathrm{H}$, Bushaqer $\mathrm{N}$, Ayyoub $\mathrm{H}$, et al. Antimullerian hormone (AMH) level and IVF/ICSI cycle outcome in expected poor responders. Middle East Fertility Society Journal. 2018; 23(3): 246-250, doi: 10.1016/j. mefs.2017.10.007.

12. Jiang $X$, Yan J, Sheng $Y$, et al. Low anti-Müllerian hormone concentration is associated with increased risk of embryonic aneuploidy in women of advanced age. Reprod Biomed Online. 2018; 37(2): 178-183, doi: 10.1016/j.rbmo.2018.04.039, indexed in Pubmed: 29724534.

13. Zhang J, Liu H, Mao X, et al. Effect of endometrial thickness on birthweight in frozen embryo transfer cycles: an analysis including 6181 singleton newborns. Hum Reprod. 2019; 34(9): 1707-1715, doi: 10.1093/humrep/dez103, indexed in Pubmed: 31398256.

14. Wei D, Liu JY, Sun Y, et al. Frozen versus fresh single blastocyst transfer in ovulatory women: a multicentre, randomised controlled trial. Lancet. 2019; 393(10178): 1310-1318, doi: 10.1016/S0140-6736(18)32843-5, indexed in Pubmed: 30827784

15. Wu T, Yin B, ZhuY, et al. Molecular cytogenetic analysis of early spontaneous abortions conceived from varying assisted reproductive technology procedures. Mol Cytogenet. 2016; 9: 79, doi: 10.1186/s13039-016-0284-2, indexed in Pubmed: 27752285.

16. Lanasa MC, HoggeWA, KubikCJ, et al. A novel X chromosome-linked genetic cause of recurrent spontaneous abortion. Am J Obstet Gynecol. 2001; 185(3): 563-568, doi: 10.1067/mob.2001.117670, indexed in Pubmed: 11568778.

17. Cheng HH, Ou CY, Tsai CC, et al. Chromosome distribution of early miscarriages with present or absent embryos: female predominance. J Assist Reprod Genet. 2014; 31(8): 1059-1064, doi: 10.1007/s10815-014-0261-9, indexed in Pubmed: 24879519.

18. Rodgers CS, Creasy MR, Fitchett M, et al. Solid tissue culture for cytogenetic analysis: a collaborative survey for the Association of Clinical Cytogeneticists. J Clin Pathol. 1996; 49(8): 638-641, doi: 10.1136/jcp.49.8.638, indexed in Pubmed: 8881913.

19. Bell K, Deerlin PV, Haddad B, et al. Cytogenetic diagnosis of "normal $46, X X$ " karyotypes in spontaneous abortions frequently may be misleading. Fertility and Sterility. 1999; 71(2): 334-341, doi: 10.1016/s00150282(98)00445-2.

20. Bingol B, Abike F, Gedikbasi A, et al. Comparison of chromosomal abnormality rates in ICSI for non-male factor and spontaneous conception. J Assist Reprod Genet. 2012; 29(1): 25-30, doi: 10.1007/s10815-0119646-1, indexed in Pubmed: 22038381.

21. Zhang T, Sun $Y$, Chen $Z$, et al. Traditional and molecular chromosomal abnormality analysis of products of conception in spontaneous and recurrent miscarriage. BJOG. 2018; 125(4): 414-420, doi: 10.1111/14710528.15052, indexed in Pubmed: 29220118.

22. HuY, Chen $X$, Chen LL, et al. Comparative genomic hybridization analysis of spontaneous abortion. Int J Gynaecol Obstet. 2006; 92(1): 52-57, doi: 10.1016/j.ijgo.2005.09.013, indexed in Pubmed: 16263126.

23. Robbins SM, Thimm MA, Valle D, et al. Genetic diagnosis in first or second trimester pregnancy loss using exome sequencing: a systematic review of human essential genes. J Assist Reprod Genet. 2019; 36(8): 1539-1548, doi: 10.1007/s10815-019-01499-6, indexed in Pubmed: 31273585.

24. Chang J, Boulet SL, Jeng G, et al. Outcomes of in vitro fertilization with preimplantation genetic diagnosis: an analysis of the United States Assisted Reproductive Technology Surveillance Data, 2011-2012. Fertil Steril. 2016; 105(2): 394-400, doi: 10.1016/j.fertnstert.2015.10.018, indexed in Pubmed: 26551441.

25. Rodriguez-Purata J, Lee J, Whitehouse M, et al. Embryo selection versus natural selection: how do outcomes of comprehensive chromosome screening of blastocysts compare with the analysis of products of conception from early pregnancy loss (dilation and curettage) among an assisted reproductive technology population? Fertil Steril. 2015; 104(6): 1460-14666.e1, doi: 10.1016/j.fertnstert.2015.08.007, indexed in Pubmed: 26361205. 